\title{
APPORTS DES IMAGES LANDSAT Á LA DYNAMIQUE DE L'OCCUPATION DU SOL ET Á L'ANALYSE DES CONFLITS D'USAGE DANS LE DÉPARTEMENT DE DIANRA AU NORD DE LA CÔTE D'IVOIRE
}

\author{
KONAN Kouadio Eugène, AMANI Konan Romaric Parfait
}

Institut de géographie tropicale (IGT), Université Félix Houphouët-Boigny (Côte d'Ivoire)

Kouadio Eugene KONAN <enzokkeugene@yahoo.fr>

\begin{abstract}
Résumé
Localisé dans la zone d'extension pastorale au nord de la Côte d'Ivoire, le département de Dianra subit une recomposition des formes spatiales qui structurent son territoire. L'enjeu majeur de l'analyse des mutations socioécologiques dans ce contexte, est l'identification des différents types de conflits d'usage liés à la dynamique de l'occupation du sol, obtenue à partir des techniques de télédétection. L'étude a en partie été effectuée sur la base de l'analyse d'images satellite Landsat réalisées en 1986, 2001 et 2016. Elle s'appuie également sur une recherche bibliographique ainsi que sur la mobilisation de données collectées lors d'enquêtes de terrain (du 05 févier au 05 mars 2016), dans le cadre d'une étude visant à comprendre l'évolution des règles, des normes et des comportements liés à l'usage des ressources naturelles. Les données recueillies ont permis de séquencer cette évolution en deux périodes. La première, allant de 1986 à 2001, est caractérisée par une abondance des ressources ligneuses, avec $70 \%$ de savane (savane arbustive et savane arborée) en 1986 et $16 \%$ de forêt dense sèche et de forêt-galerie. Cette période est marquée par une gestion communautaire des ressources naturelles. La deuxième période, allant de 2001 à 2016, est caractérisée par la diminution des ressources ligneuses, avec $37 \%$ de savane (savane arbustive et savane arborée) et $2 \%$ de forêt dense sèche et de forêt- galerie. La dynamique de l'occupation du sol conduit au rétrécissement des pâtures naturelles et au morcellement de l'espace. Cela engendre des conflits d'usage de plus en plus fréquents entre agriculteurs d'anacarde et éleveurs, qui ont des visions antagonistes de l'usage de l'espace, ainsi que des conflits hégémoniques entre villages pour le contrôle des espaces.
\end{abstract}

Mots-clés : Landsat, Dianra, Côte d'Ivoire, occupation du sol, anacarde, conflits d'usage

\begin{abstract}
Located in the pastoral extension zone in the north of Côte d'Ivoire, the Dianra department is undergoing a recomposition of the spatial forms that structure its territory. The major challenge of analysing socio-ecological changes in this context is to identify the different types of use conflicts linked to the dynamics of land use, obtained using remote sensing techniques. The study was partly based on the analysis of Landsat satellite images taken in 1986, 2001 and 2016. It is also based on bibliographical research as well as on the mobilisation of data collected during field surveys (from 05 February to 05 March 2016), as part of a study aimed at understanding the evolution of rules, norms and behaviours related to the use of natural resources. The data collected made it possible to sequence this evolution into two periods. The first, from 1986 to 2001, is characterised by an abundance of woody resources, with 70\% savannah (shrub savannah and tree savannah) in 1986 and $16 \%$ dense dry forest and gallery forest. This period is marked by community management of natural resources. The second period, from 2001 to 2016, is characterised by a decrease in woody resources, with $37 \%$ savannah (shrub savannah and tree savannah) and $2 \%$ dense dry forest and gallery forest. The dynamics of land use lead to the shrinking of natural pastures and the fragmentation of space. This leads to increasingly frequent conflicts of use between cashew farmers and herders, who have antagonistic visions of the use of space, as well as hegemonic conflicts between villages for the control of space.
\end{abstract}

Keywords: Landsat, Dianra, Côte d'Ivoire, land cover, cashew, conflicts use.

\section{1-Introduction}

Depuis les années 1970, le nord de la Côte d'Ivoire connaît une recomposition sociale et une nouvelle structuration spatiale de son territoire. Ce phénomène est dû à l'installation d'éleveurs transhumants ayant fui les régions sahéliennes (Mali, Burkina Faso) à cause de la grande sécheresse qui sévissait dans leurs régions d'origine (Diallo et al., 2011).

Les autorités ivoiriennes ont profité de la survenue de cette crise climatique pour développer une politique pastorale encourageant la sédentarisation des éleveurs transhumants peuls (Burkinabés et Maliens) en vue de pallier la grande dépendance de la Côte d'Ivoire en protéine animale ((Brou et Charléard, 2007, Corniaux, et al, 2012).
Cette politique volontariste de l'État ivoirien a abouti à un essor fulgurant de l'élevage et à l'émergence de l'agropastoralisme dans la partie septentrionale du pays.

Ce nouveau contexte social a entraîné de nouvelles règles d'occupation spatiale et la mise en place de comités de résolution des conflits entre les différents acteurs. Les autochtones (agriculteurs) et les allochtones (éleveurs) avaient en effet des visions différentes de la pratique de l'espace et de l'usage des ressources naturelles (Dugue et al., 2004).

Cette reconfiguration majeure des dynamiques qui se sont exercées a fortement marqué le département de Dianra. La création de barrages pour l'abreuvage des troupeaux, et de pâturages, sont des exemples de ces recompositions des usages de l'espace. L'intensification des activités agricoles et de l'élevage a suscité des conflits entre ces groupes d'acteurs à l'intérieur d'espaces de plus en plus disputés. 
Face à la transformation du contexte agricole, les vergers d'anacardiers (cf. Figure 1) se sont multiplié, transformant le paysage du département de Dianra. A la suite de cette conversion de l'espace, les systèmes pastoraux, qui sont essentiellement de type extensif, se trouvent confrontés à des difficultés pour disposer d'espaces suffisamment vastes permettant d'entretenir un cheptel dont les effectifs ont considérablement augmenté depuis le début des années 1970 (Landais, 1994 ; Sokouri et al., 2009)

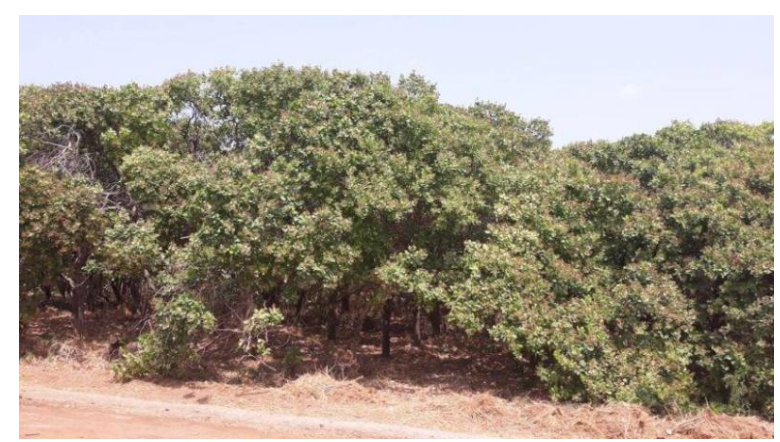

Figure 1 : Verger d'anacardiers à Dianra

Ce contexte de raréfaction de l'espace alloué à l'activité pastorale suscite des changements institutionnels qui s'articulent autour de règles d'accès à la terre, et de comportements et de normes régissant le système d'occupation de l'espace en vue de sécuriser l'occupation du sol et l'usage des ressources naturelles.

Malgré ces dynamiques sociales, l'usage des ressources s'intensifie et l'occupation de l'espace s'accélère dans le département de Dianra.
Au-delà des compétitions qui s'organisent entre agriculteurs et éleveurs pour l'accès aux ressources, les conflits se multiplient entre des groupes qui revendiquent les mêmes droits sur des ressources dont ils sont tributaires pour leur survie. Quelles sont alors les caractéristiques des conflits d'usage liés à la dynamique de l'occupation de l'espace dans le département de Dianra dans ce contexte où les enjeux concernant sa maitrise se multiplient ? Pour répondre à cette question, cette étude se fixe comme objectif d'analyser les conflits d'usage issus de la dynamique de l'occupation du sol s'étant déroulés entre 1986 et 2016 dans ce département. II s'agit d'une part d'identifier, à l'aide des techniques de télédétection, les types d'occupation de l'espace du département de Dianra en 1986, 2001 et 2016, et d'autre part de déterminer les types de conflits d'usage liés aux changements de types d'occupation du sol de 1986 à 2016.

\section{2-Présentation de la zone d'étude}

Le département de Dianra est situé dans la région du Béré, dans le nord de la Côte d'Ivoire (cf. Figure 2), précisément entre $6^{\circ} 0^{\prime}$ et $7^{\circ} 10^{\prime}$ de longitude ouest et $8^{\circ} 30^{\prime}$ et $9^{\circ} 10^{\prime}$ de latitude nord. II s'étend sur une superficie de $3073,95 \mathrm{~km}^{2}$. II est composé de deux souspréfectures : la sous-préfecture de Dianra au nord, cheflieu de département ayant une superficie de $1581 \mathrm{~km}^{2}$, et la sous-préfecture de Dianra village situé au sud du département, doté d'une superficie de $1494 \mathrm{~km}^{2}$. Le département de Dianra est limité au nord par le département de Boundiali, au sud par le département de Mankono, à l'ouest par le département de Kani, au nordest par le département de Korhogo et au sud-est par le département Dikodougou. 


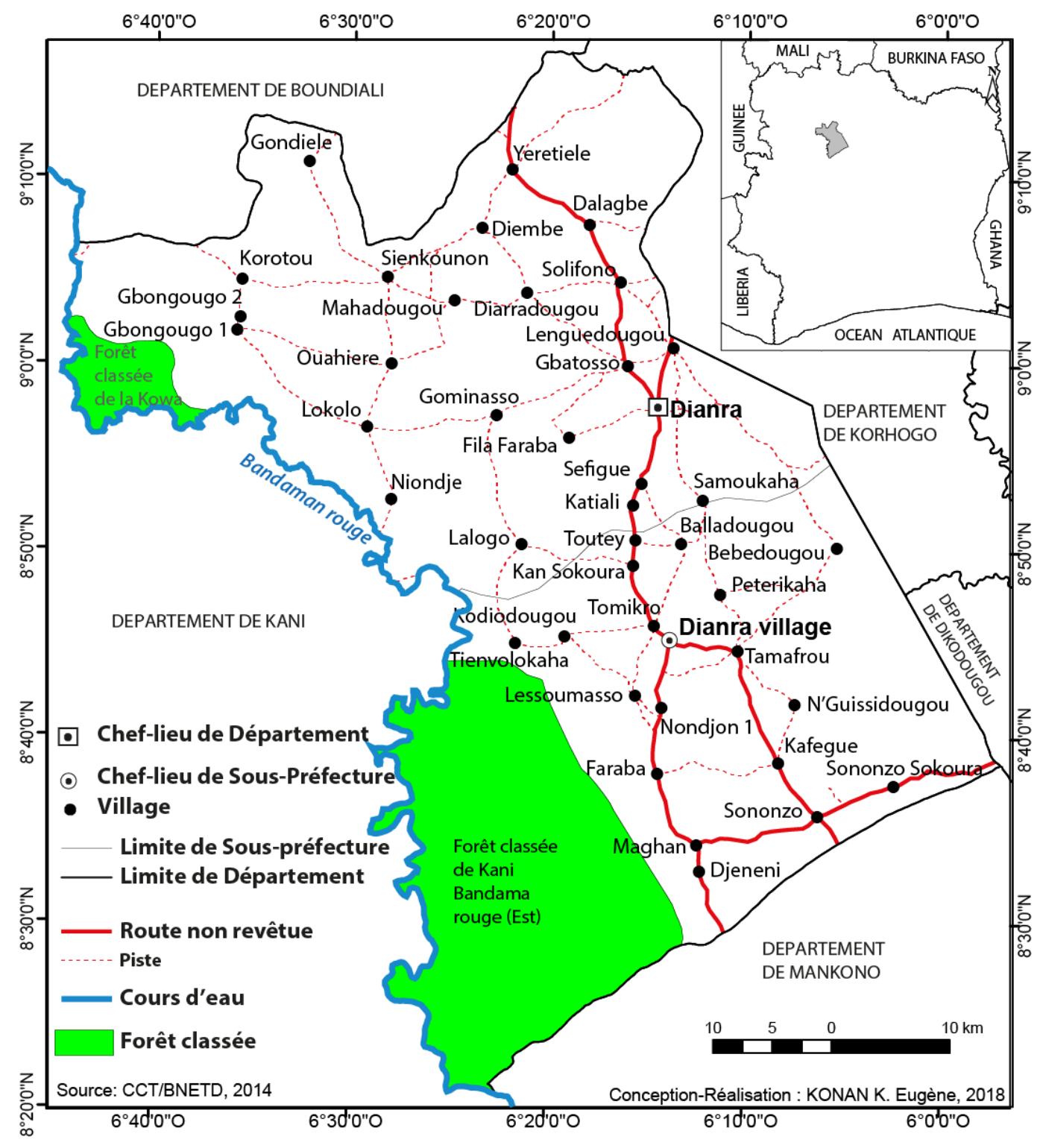

Figure 2 : Présentation du département de Dianra : [Source : BNETD, 2014]

Situé dans une zone agroécologique dite zone soudanoguinéenne, le département de Dianra est caractérisé par un climat de type "soudanien », rythmé par deux saisons distinctes (Avenard et al, 1971). La saison sèche est en général marquée par le phénomène de l'harmattan, vent chaud et sec soufflant sur l'Afrique occidentale du mois de novembre au mois de mai (Mahop, 1983). Les températures varient alors de $25^{\circ} \mathrm{C}$ à $31^{\circ} \mathrm{C}$, avec une moyenne de $28^{\circ} \mathrm{C}$. La saison pluvieuse se situe entre le mois de mai et le mois d'août. Elle est caractérisée par une pluviométrie moyenne annuelle variant entre $0 \mathrm{~mm}$ en décembre et $288 \mathrm{~mm}$ en août. Le département de Dianra est drainé par le Bandama rouge et ses affluents. On y trouve aussi de nombreuses rivières et marigots qui tarissent lors de la saison sèche. La végétation est de type savane sub-soudanienne. Toutes les formations savanicoles sont présentes, de la savane boisée à la savane herbeuse. La forêt caractéristique de la région est la forêt dense sèche, que l'on retrouve essentiellement sur les sommets des interfluves et les galeries forestières. Cette forêt est fortement dégradée par l'action de l'homme à travers les exploitations agricoles

La population du département est estimée à 95579 habitants selon le recensement général de la population et de l'habitat (Institut national de la statistique, 2014), ce qui représente $25 \%$ de la région du Béré et moins de 1 $\%$ de la population nationale. Ce département connaît d'importants flux migratoires des populations des régions voisines et des pays limitrophes du nord de la Côte d'Ivoire (Mali, Burkina Faso). Outre l'immigration, la croissance naturelle participe à l'augmentation de la population. Le taux d'accroissement naturel de 1,6\% explique l'augmentation de la population. La population du département est majoritairement rurale et se compose de $53 \%$ d'hommes et $47 \%$ de femmes (INS, 2014).

\section{Méthodes}

\subsection{Données}

La réalisation de cette étude s'appuie sur un matériel, composé de données satellitaires et cartographiques, ainsi que de logiciels.

La mission de terrain a été réalisée à l'aide d'un GPS pour la géolocalisation des traces humaines et des zones d'apprentissage pour la phase de classification des images, d'un appareil photo numérique pour les prises de vue de certains sites de contrôle, et d'un dictaphone pour l'enregistrement des entretiens. 
Les données cartographiques et satellitaires utilisées sont constituées d'une carte topographique du département à l'échelle 1 / 500000 datant de 1994, obtenue à la Société de développement des forêts (SODEFOR), d'une carte de la végétation à l'échelle 1 500000 élaborée par l'ORSTOM en 1968, obtenue à l'Institut de recherche et de développement (IRD), et de six images satellitaires. Ces images sont composées de deux images Landsat 5 (TM) de résolution $30 \mathrm{~m}$, datant du 7/01/1986 et du 14/01/1986, de deux images Landsat $7(\mathrm{ETM}+)$ de résolution $30 \mathrm{~m}$, datant du 16/01/2001 et du 23/01/2001, et de deux images Landsat 8 (OLI/TIRS) de résolution $30 \mathrm{~m}$, datant du 04/02/2016 et du 27/02/2016 toutes des scènes 197-054 et 198-054 (tableau 1) réalisées en saison sèche.

Le choix de la saisonnalité identique pour les trois images (janvier / février) est lié à la non-présence des nuages à cette période de l'année, qui permet a priori de mieux comparer les traitements effectués.

La Figure 3 présente des extraits d'images issus des compositions colorées effectuées. Les compositions colorées sur les images Landsat 5 (TM) et Landsat 7 (ETM+) ont été obtenues en affectant la bande 2 dans la gamme du rouge, la bande 4 dans la gamme du vert et la bande 6 dans la gamme du bleu. La composition colorée de l'image Landsat 8 (OLI/TIRS) est issue d'une combinaison établie avec la bande 3 dans la gamme du rouge, la bande 5 dans la gamme du vert et la bande 7 dans la gamme du bleu. Les canaux choisis permettent une meilleure discrimination des entités spatiales étudiées. Ils mettent surtout en exergue les formations ligneuses et permettent visuellement de comparer les trois images grâce à l'usage des mêmes couleurs et teintes.

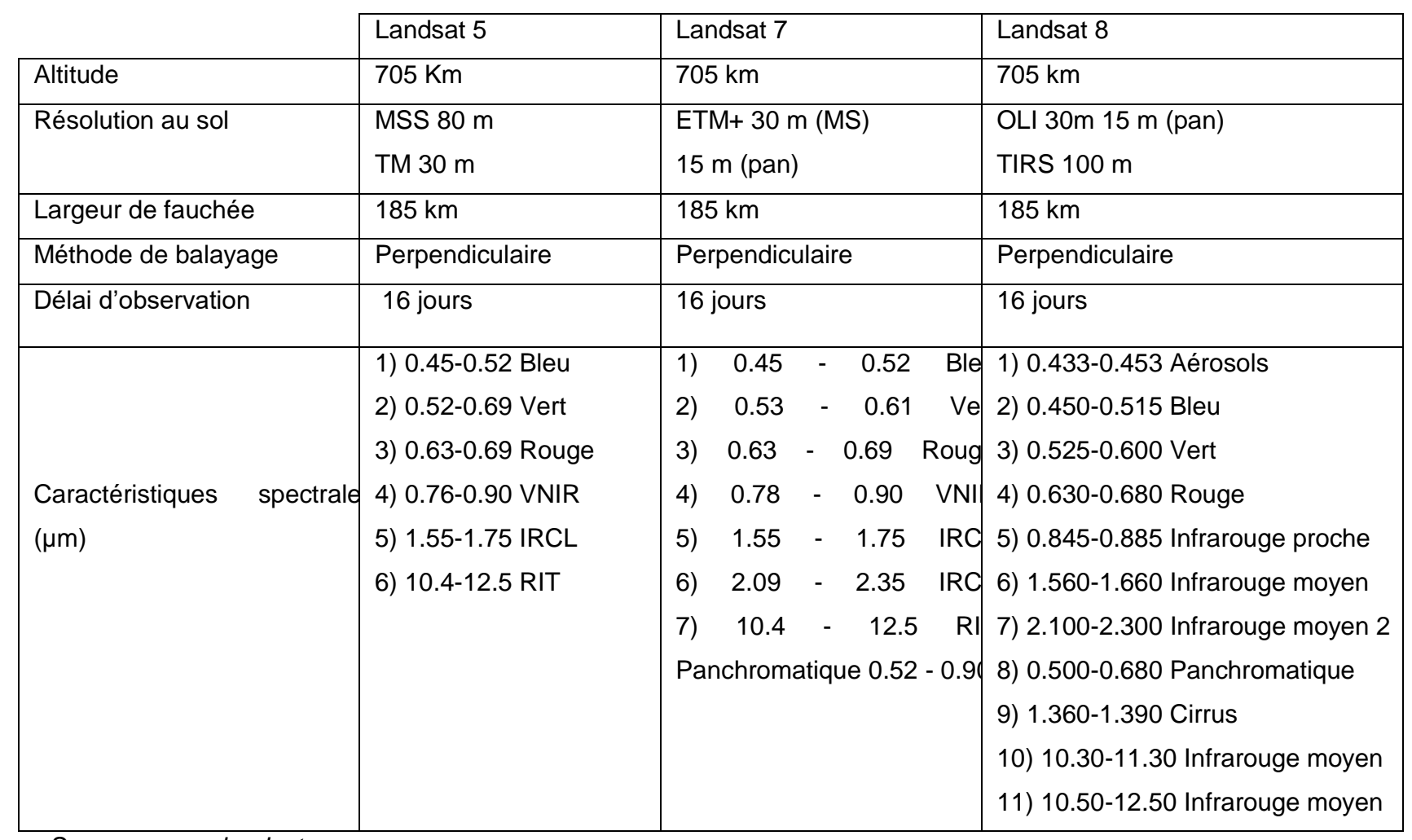

Source : www. landsat.org

Tableau 1: Caractéristiques des bandes spectrales des images satellites Landsat utilisées 


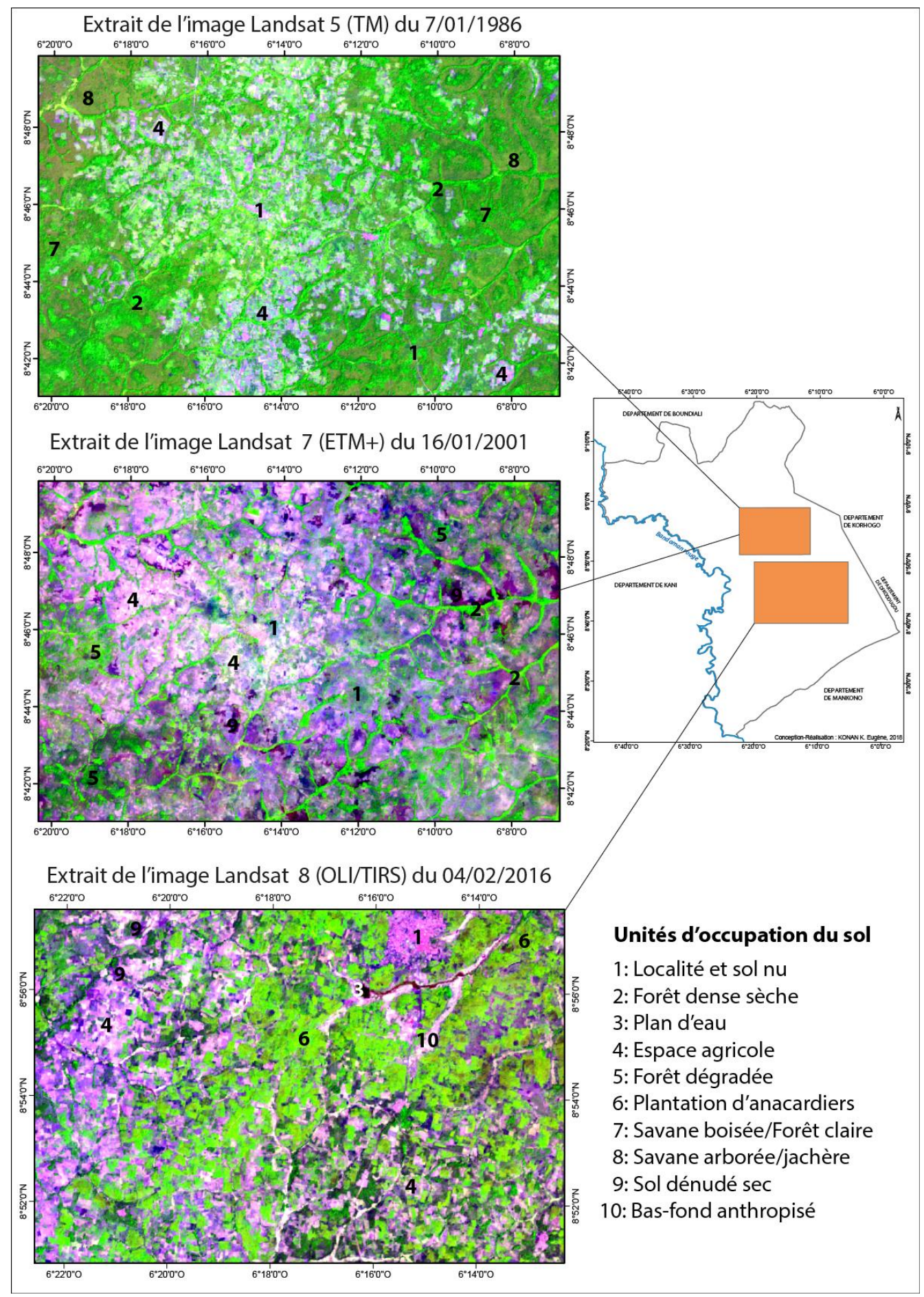

Figure 3 : Extraits d'images Landsat 5 et 7 (Rouge = bande 2, Vert = bande 4, Bleu = bande 6$)$ et Landsat $8($ Rouge $=$ bande 3 , Vert $=$ bande 5 , Bleu $=$ bande 7$)$

\subsection{Techniques de collecte des données}

Dans cette étude, les techniques de collecte d'information utilisées sont la recherche documentaire et l'enquête de terrain.

La recherche documentaire a permis de connaître l'état d'avancement des travaux effectués sur la thématique. Ainsi des articles et revues scientifiques, des thèses, mémoires et des documents divers ont été consultés dans des bibliothèques et sur internet («Google scholar » et «Research 4 life»).
Une enquête effectuée sur le terrain du 05 févier au 05 mars 2016 a permis de procéder à l'observation sur le terrain, et à 37 entretiens avec les autorités administratives et les chefs de terre (les Talafolos). L'observation sur le terrain a consisté à effectuer des relevés des types d'affectation au sol à l'aide d'un GPS afin d'avoir une idée de l'occupation de l'espace du site d'étude. 


\subsection{Traitement et analyse des données}

Le suivi de la dynamique écologique est d'abord analysé du point de vue de la dynamique du couvert végétal, à partir de méthodes de télédétection. Ainsi, un certain nombre de techniques de télédétection sont utilisées en vue de mettre en évidence les changements de l'état de la surface. Pour produire des cartes d'occupation du sol, trois phases sont admises : la phase de prétraitement des images, la phase de traitement des images, et l'utilisation d'un système d'information géographique (SIG).

\subsubsection{Prétraitement des images}

Les prétraitements d'images sont un ensemble d'opérations qui ont pour but d'augmenter la lisibilité des données pour faciliter leur interprétation. Ils se résument dans cette étude au mosaïquage, à l'amélioration des contrastes des images et à l'extraction de la zone d'étude. II faut souligner que les images obtenues ont déjà été corrigées radiométriquement et atmosphériquement.

La zone d'étude étant couverte par deux scènes d'images (197-54 et 198-54), il s'est avéré nécessaire d'assembler ces scènes avant le traitement. Le mosaïquage consiste à juxtaposer les différentes bandes des scènes qui sont identiques. Cette technique permet de former une seule image (Jobin, 2007).

\subsubsection{Traitement des images}

La phase de traitement des images consiste en la définition des classes d'occupation du sol, en la cartographie de l'occupation du sol et en la validation de la classification (évaluation de la précision).

\subsubsection{Définition des classes d'occupation du sol}

Les différentes classes d'occupation du sol retenues dans cette étude sont les espaces de forêt, les savanes, les surfaces agricoles, les surfaces d'eau, les habitats et sols nus, les surfaces dénudées. La définition de ces classes a ét effectuée à partir d'images issues de compositions colorées en vraies couleurs (trois bandes qui correspondent au rouge, au vert et au bleu) qui permettent une bonne discrimination spectrale des affectations au sol, favorisant l'identification des entités à cartographier sur l'image satellitaire. L'image de base choisie pour cette opération est celle de 2016, car elle est la plus récente. Aussi, les sites échantillonnés (276 points) géolocalisés pendant l'observation de terrain ontils été importants dans le repérage des éléments sur les images.

\subsubsection{Cartographie de l'occupation $d u$ sol par télédétection}

La cartographie de l'occupation du sol s'est faite par le biais d'une classification selon les classes d'occupation du sol définies. La technique utilisée ici est la classification dirigée ou supervisée, qui consiste en une hiérarchisation par étapes successives ou classification hiérarchique pseudo-dirigée (Fotsing, 2009). L'algorithme utilisé dans cette classification est celui du maximum de vraisemblance, lequel repose sur la règle de Bayes et permet de calculer pour chaque pixel sa probabilité d'appartenir à une classe plutôt qu'à une autre. Le pixel est affecté à la classe dont la probabilité d'appartenance est la plus élevée.

\subsubsection{Validation de la classification (évaluation de la précision)}

Deux indicateurs de précision ont été calculés et une campagne de terrain a été effectuée en vue de la validation des classifications:
- l'analyse de la séparabilité des classes représente des tests statistiques permettant de mesurer la capacité d'un paramètre à discriminer deux classes différentes ;

- la matrice de confusion est une technique qui permet de confronter deux classifications: celle des sites d'entraînement et celle des sites de contrôle. En effet, la matrice permet de calculer la précision globale (OA : Overall Accuracy) et l'indice de Kappa (K) (Dusseux, 2014). Généralement, il est établi que les classifications ayant des valeurs de $K$ supérieures à 0.8 sont excellentes, celles ayant des valeurs de $K$ comprises entre 0.6 et 0.8 sont acceptables et que celles ayant des valeurs de $\mathrm{K}$ comprise entre 0.4 et 0.6 montrent une faible précision.

- la campagne de terrain a permis de confronter la conformité du contenu thématique des images classifiées à la réalité de terrain. Elle a consisté en l'observation minutieuse des différentes classes d'occupation du sol sur des sites présélectionnés.

\subsubsection{Amélioration de la classification}

Les résultats bruts issus de la classification ont été soumis à un filtrage post-classification pour nettoyer l'image de classification afin de la faire correspondre aux exigences d'UMC (unité minimale de cartographie) et ainsi de réduire les erreurs tout en éliminant les pixels isolés. L'UMC adoptée pour produire la carte d'occupation du sol est de 0,1 ha, conformément au nouveau code forestier de Côte d'lvoire qui fixe la superficie minimale des forêts à 0,1 ha. La méthodologie adoptée pour le post-traitement se résume en deux passages : le passage d'un filtre convolutif majoritaire de dimension $3 \times 3$ pixels permettant de réduire le bruit, et celui d'un filtre de tamisage d'une taille de 10 pixels, correspondant exactement à $9000 \mathrm{~m}^{2}$, soit 0,9 hectares. Une fois le filtrage terminé, les images ont été converties en vecteur et exportées dans un environnement SIG, plus précisément dans ArcGis 10.2.2.

\subsubsection{Utilisation d'un système d'information} géographique (SIG)

Les données issues du traitement des images ont été exportées dans un environnement SIG pour créer une base de données. Cette base a été renforcée par l'ajout de données cartographiques (localités, routes, réseau hydrographique et limites). Le SIG peut être défini comme un outil informatique permettant de stocker, de gérer, de traiter et de représenter l'information géographique dans le but d'une prise de décision (Konan, 2008). L'exploitation de la base de données a consisté à faire des opérations de requêtes sur une ou plusieurs données de la base dans le but de rechercher l'information ou de produire de nouvelles données cartographiques, graphiques ou statistiques. Deux logiciels ont été utilisés pour le traitement des données: Envi 5.1 pour le traitement des images satellitaires, et ArcGis 10.2.2 pour les applications SIG et les rédactions cartographiques.

\section{4-Résultats}

\section{1- Signature des différents types d'occupation du sol sur les images avec une validation terrain}

Les types d'occupations du sol retenus pour la classification ont fait l'objet d'une description afin de mieux les cerner. Ils sont présentés dans le Tableau 2. 


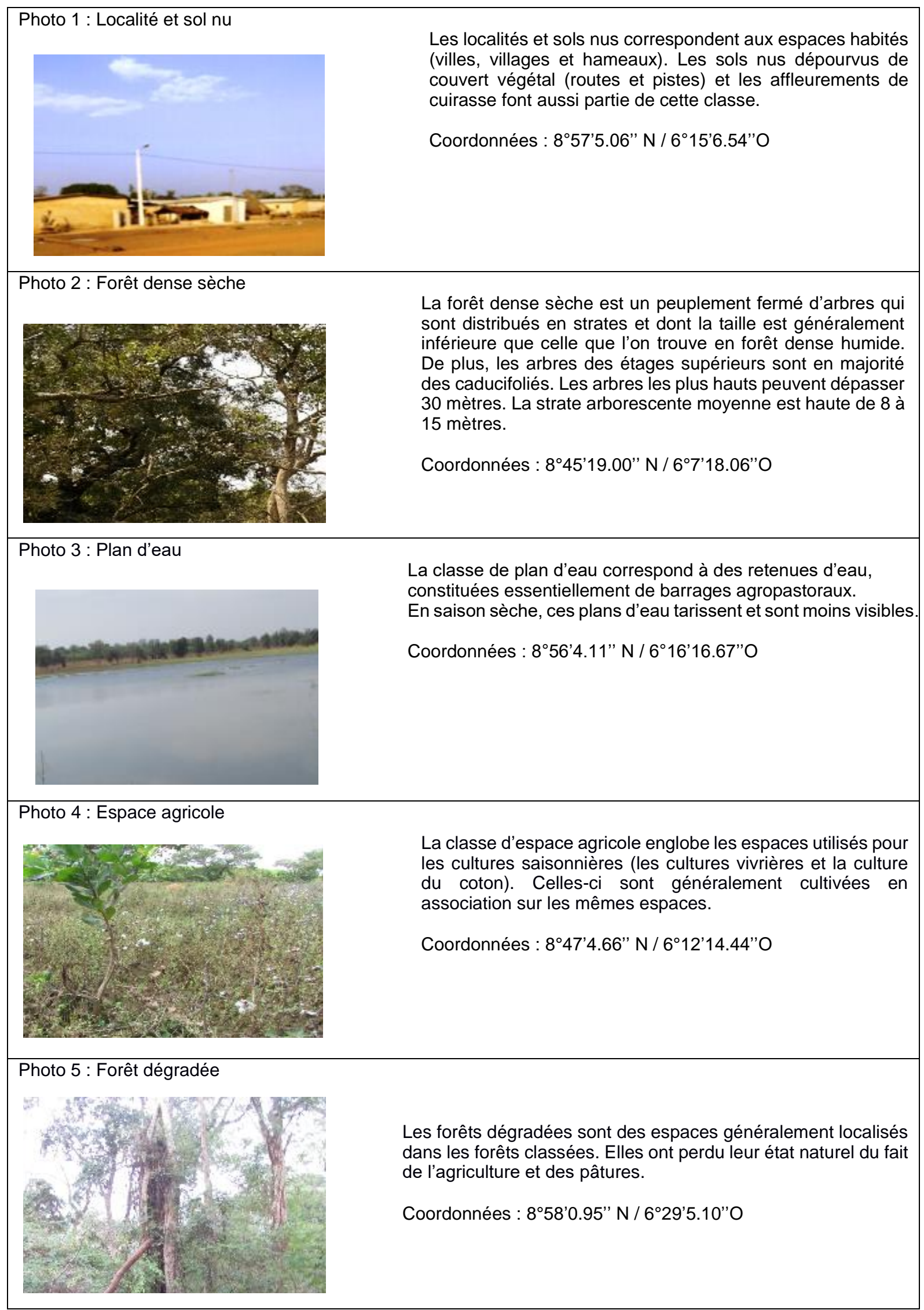




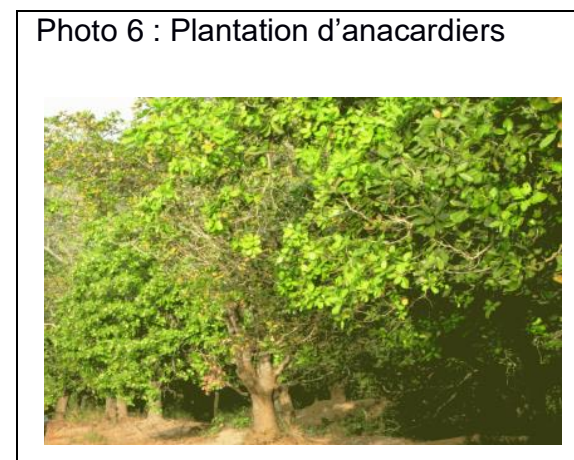

Les plantations d'anacardiers sont composées d'arbres fruitiers dont la taille moyenne est comprise entre 6 et 8 mètres de hauteur. Ils sont cultivés par les populations pour son fruit, l'anacarde.

Coordonnées : 858'11.63"' N / 6¹5’35.48”O

Photo.7 : Savane boisée/Forêt claire

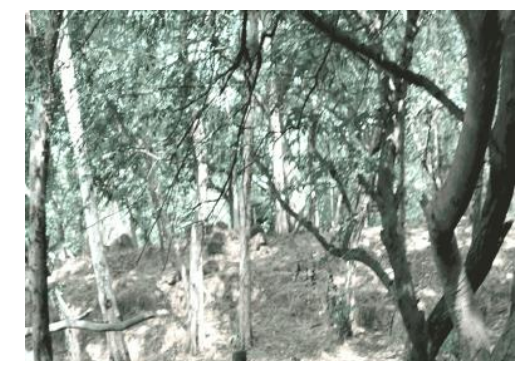

Photo. 8 : Savane arborée/jachère

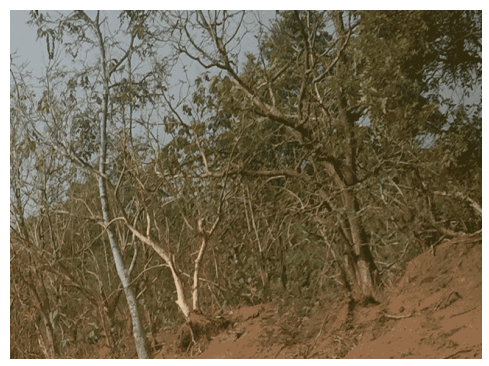

Photo.9 : Sol dénudé sec

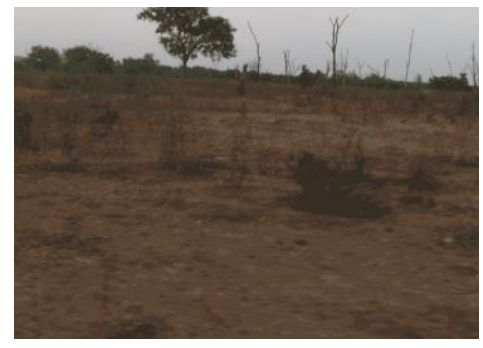

Photo.10 : Bas-fond anthropisé

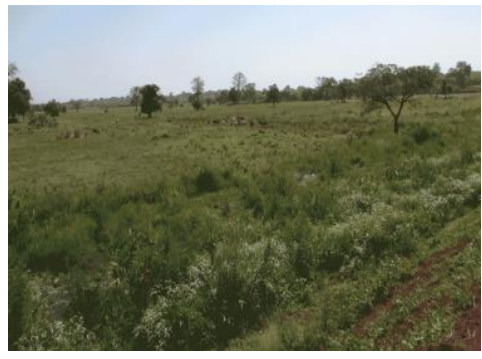

La classe associant savane boisée et forêt claire correspond à des peuplements ligneux ouverts, aux cimes plus ou moins jointives laissant largement filtrer la lumière. Au sol, le tapis graminé est relativement peu dense.

Coordonnées : 90'46.35" N / 6³8'6.12"O
Cette classe est constituée d'arbres dont la hauteur est comprise entre 6 et 8 mètres.

Coordonnées : 90'5.19” N / 641'43.42”O

Cette classe correspond au sol dénudé sec dépourvu de végétation. II s'agit principalement d'espaces défrichés pour l'agriculture.

Coordonnées : 8²8'3.02" N / 6³'25.80”O

Les bas-fonds sont des unités paysagères les plus basses de la toposéquence. Ils constituent des zones de rencontre et d'organisation des eaux descendues des versants. Ces sites sont généralement utilisés par les agriculteurs pour l'implantation des rizières.

Coordonnées : 855’4.00” N / 6¹4’57.64”O

Tableau 2 : Description des différents types d'occupation du sol retenus 


\section{2- Analyse de la matrice de confusion de 1986 à 2016}

La classification de l'image de 1986 donne 93,50\% de pixels bien classés avec un indice de Kappa de 0,92 (cf Tableau 3). Cette classification comporte trois thèmes qui posent des problèmes de répartition statistique des pixels. D'abord, une confusion importante est relevée entre les espaces en eau et les forêts denses sèches avec $69,3 \%$ de pixels bien classés et de $28,22 \%$ de pixels classés en forêts. Cela est dû à la présence des cours d'eau intermittents qui serpentent le long des galeries forestières dans un contexte de forte présence de couverture boisée. Les confusions se produisent également entre les savanes boisées et forêts claires avec $85,40 \%$ de pixels bien classés et $12,87 \%$ classés en forêts denses sèches. Les conséquences peuvent être considérées comme négligeables puisque ces erreurs concernent des espaces forestiers souvent considérés comme similaires sur le terrain et très difficiles à identifier (Oszwald et al, 2010).

\begin{tabular}{l|c|c|c|c|c|c|c}
\hline Classes & 1 & 2 & 3 & 4 & 5 & 6 & 7 \\
\hline 1.Localités & $\mathbf{8 9 , 3 7}$ & 0 & 0 & 1,85 & 0 & 0 & 0 \\
\hline 2.Forêt dense sèche & 0 & $\mathbf{9 9 , 6}$ & 0 & 0 & 0,08 & 12,9 & 0 \\
\hline 3.Espaces en eau & 0 & 0 & $\mathbf{6 9 , 3 3}$ & 0 & 0 & 0 & 0 \\
\hline 4.Espaces agricoles & 4,66 & 0 & 0 & $\mathbf{8 9 , 3 4}$ & 0,29 & 0,14 & 0 \\
\hline 5.Savane arborée & 5,97 & 0 & 28,22 & 8,16 & $\mathbf{9 5 , 9 1}$ & 1,95 & 0,39 \\
\hline 6.Savane boisée & 0 & 0,4 & 0 & 0,12 & 2,38 & $\mathbf{8 5}$ & 0 \\
\hline 7.Sols nus & 0 & 0 & 2,45 & 0,52 & 1,34 & 0 & $\mathbf{9 9 , 6 1}$ \\
\hline Total & 100 & 100 & 100 & 100 & 100 & 100 & 100 \\
\hline \multicolumn{7}{c}{ (Source : Landsat, 1986) } \\
\hline \multicolumn{7}{c}{ Précision globale : 93,50 \%. Coefficient Kappa (K) : 0, 92 }
\end{tabular}

Tableau 3 : Matrice de confusion de la classification de l'image de 1986 (en \%)

La classification de l'image de 2001 présente un pourcentage de pixels bien classés de $96,18 \%$, avec un coefficient Kappa de 0.94 (cf. Tableau 4). Les erreurs de classifications sont surtout induites par la confusion entre les classes de forêts denses sèches et les plantations d'anacardiers: $55 \%$ des pixels correspondant aux plantations d'anacardiers sont bien classés, contre $32,2 \%$ classés en forêt dense sèche. Cette confusion est liée à la présence d'une strate arborescente sans ouverture majeure du couvert (plus de $80 \%$ ) dans les deux classes. Le même phénomène peut être observé pour le thème lié aux plantations d'anacardiers et aux savanes boisées. La principale confusion a donc lieu entre les différentes parcelles à couvert végétal où sont présents des vergers comportant de jeunes plants d'anacardier qui, du point de vue radiométrique, se comportent comme des surfaces de savane. Cette confusion s'explique par le fait qu'en 2001, les plantations d'anacardiers ne s'étendaient que sur de petites surfaces. II est difficile de les distinguer avec une grande précision à partir de l'image satellitaire Landsat ETM+ à 30 mètres de résolution. Toutefois, la forte précision de classification globale et du coefficient Kappa confirme la justesse de la classification.

\begin{tabular}{|c|c|c|c|c|c|c|c|c|}
\hline Classes & 1 & 2 & 3 & 4 & 5 & 6 & 7 & 8 \\
\hline 1.Localités & 93,49 & 0 & 0 & 3,64 & 0 & 0,37 & 0 & 3,52 \\
\hline 2.Forêt dense sèche & 0 & 96,69 & 0,01 & 0 & 0,88 & 0,31 & 32,2 & 0 \\
\hline 3.Espace en eau & 0 & 0 & 97,87 & 0 & 0 & 0 & 0 & 0 \\
\hline 4.Espaces agricoles & 6,14 & 0 & 0 & 94,91 & 0 & 0,09 & 0 & 0 \\
\hline 5.Savane arborée & 0 & 0 & 2,13 & 1,34 & 97,47 & 1,05 & 1,88 & 0,99 \\
\hline 6.Savane boisée & 0,37 & 3,31 & 0 & 0,09 & 1,05 & 97,08 & 9,89 & 0,26 \\
\hline 7.Plantations d'anacardiers & 0 & 0 & 0 & 0 & 0 & 1,16 & 55,93 & 0 \\
\hline 8.Sols nus & 0 & 0 & 0 & 0 & 1,49 & 0 & 0 & 98,84 \\
\hline Total & 100 & 100 & 100 & 100 & 100 & 100 & 100 & 100 \\
\hline
\end{tabular}

(Source : Landsat, 2001)

Précision globale : 93,50. Coefficient Kappa $(K): 0,94$

Tableau 4 : Matrice de confusion : classification de l'image de 2001 (en \%)

Les résultats de classification obtenus en 2016 montrent une confusion entre la classe des localités et celle des sols nus $(14,01 \%)$ en faveur des sols dénudés secs. De plus, on observe une sous-détection des espaces agricoles en faveur des sols dénudés secs (cf. Tableau 5). Comme cela a déjà été observé avec les profils de 1986 et 2001, les localités et sols nus montrent des similitudes avec les espaces agricoles à cause de la mise à nu des sols pour le labour. Après les récoltes, les sols restent nus, en particulier pendant les périodes sèches allant du mois de novembre au mois de février. Cela explique les résultats observés. En revanche, les classes de plantations d'anacardiers présentent des résultats satisfaisants grâce à la maturité des plantations d'anacardiers et à l'importance des surfaces occupées $(94,45 \%$ des pixels bien classés et $2.21 \%$ classés en savane boisée). Dans l'ensemble, une précision globale de $93,94 \%$ et un indice Kappa de 0,92 ont été obtenus. 


\begin{tabular}{|c|c|c|c|c|c|c|c|c|c|c|}
\hline Classes & 1 & 2 & 3 & 4 & 5 & 6 & 7 & 8 & 9 & 10 \\
\hline 1.Localités et sols nus & 80,48 & 0 & 0 & 0,22 & 0 & 0,13 & 0 & 0,02 & 0,04 & 0,09 \\
\hline 2.Forêt dense sèche & 0 & 99,01 & 0,49 & 0,01 & 0,16 & 0 & 0,05 & 0 & 0 & 0,03 \\
\hline 3.Espaces en eau & 0 & 0 & 98,76 & 0 & 0 & 0 & 0 & 0 & 0 & 0,01 \\
\hline 4.Espaces agricoles & 1,88 & 0,08 & 0 & 91,41 & 0,35 & 0,85 & 0,51 & 4,05 & 2,3 & 0,34 \\
\hline 5.Forêt dégradée & 0 & 0,21 & 0 & 0,05 & 98,49 & 0,09 & 0,09 & 0 & 0,04 & 0 \\
\hline 6.Plantations d'anacardiers & 1,72 & 0 & 0 & 0,3 & 0,02 & 94,45 & 0,6 & 0 & 0,72 & 0,12 \\
\hline 7.Savane boisée et forêt daire & 0,44 & 0,49 & 0,12 & 0,52 & 0,87 & 2,21 & 97,14 & 0,02 & 0,41 & 0,56 \\
\hline 8.Sols nus & 14,01 & 0 & 0 & 6,14 & 0 & 0,49 & 1,05 & 94,55 & 0 & 4,42 \\
\hline 9.Bas-fonds anthropisés & 0,47 & 0 & 0 & 1,07 & 0,11 & 1,58 & 0,25 & 0 & 96,43 & 0,05 \\
\hline 10.Savane arborée et arbustive & 0,99 & 0,21 & 0,62 & 0,29 & 0 & 0,19 & 0,27 & 1,36 & 0,02 & 94,38 \\
\hline Total & 100 & 100 & 100 & 100 & 100 & 100 & 100 & 100 & 100 & 100 \\
\hline
\end{tabular}

(Source : Landsat, 2016)

Précision globale : 93,94\%. Coefficient Kappa (K) : 0,92

Tableau 5 : Matrice de confusion : classification de l'image de 2016 en pourcentage (\%)

\subsection{Dynamique spatiale de l'occupation du sol : disparition progressive des espaces naturels de 1986 à 2016}

Les termes « occupation des sols » et « utilisation (ou usage) des sols » désignent deux aspects différents de la couverture des sols. L'occupation des sols fait référence à la couverture physique de la surface terrestre, tandis que l'utilisation (ou usage) des sols se rapporte à l'usage anthropique qui en est fait, et donc plus spécifiquement à la description socio-économique des surfaces terrestres (Brown et Duh, 2004).

Les cartes d'occupation du sol (cf. Figure 4) et les différents graphiques (cf. Figure 5) permettent de mettre en évidence la dynamique spatio-temporelle des surfaces socio-écologiques.

La dynamique de l'occupation du sol s'apprécie par exemple par l'analyse de la répartition et de la proportion des surfaces des différents types d'affectations. L'analyse spatiale montre ainsi qu'en 1986, le territoire du département est occupé dans son ensemble par des espaces naturels, notamment des formations savanicoles, avec environ 215532 ha occupés, soit $71 \%$ de l'espace (savane arborée et jachère $47 \%$ et savane boisée et forêt claire $24 \%$ ). Les formations forestières correspondant à des îlots forestiers de forêts denses sèches et de galeries forestières localisés sur un axe sudouest/nord-est, couvrant une superficie de 50280 ha, soit $16 \%$ du territoire. Les espaces humanisés se situent au centre du département et sont constitués en grande partie de surfaces agricoles composées principalement de cultures vivrières et de la culture du coton, avec un taux d'occupation du territoire de $10 \%$. En 2001, l'usage agricole est resté moyennement dynamique, avec une évolution d'environ $4 \%$. Les savanes, dans leur ensemble, ont connu une augmentation de $7 \%$ de leur surface. Ce phénomène peut notamment être dû aux différentes politiques de sensibilisation et de lutte contre les feux de brousse, menées par la Société de développement des forêts (SODEFOR) dans la localité. En revanche, les forêts denses sèches et galeries forestières ont perdu la moitié, soit $8 \%$, de leur superficie. Les,populations convoitent en effet ces espaces fertiles en vue de les utiliser pour l'agriculture. Ces formations forestières sont confinées dans le sud-ouest du département. La transformation la plus importante est l'introduction de la culture de l'anacarde, qui était quasiment invisible en 1986. La superficie des vergers d'anacardiers en 2001 est estimée à environ 1117 ha, soit moins de $0,5 \%$ du territoire. Ces vergers concentrent essentiellement autour des deux principales localités que sont Dianra et Dianra village (cf. Figure 2).

Entre 2001 et 2016, d'autres types d'occupation du sol ont fait leur apparition (voir figure 4). II s'agit des bas-fonds anthropisés et des forêts dégradées, qui occupent respectivement 11195 ha, soit un peu moins de $4 \%$, et 4904 ha, soit $2 \%$ de la superficie du département. Ces mutations d'occupation du sol témoignent des effets des pressions anthropiques sur l'espace. Les forêts dégradées sont essentiellement localisées dans la forêt classée de Kani Bandama rouge située à l'ouest du département (cf. Figure 2). Quant aux bas-fonds anthropisés, leur apparition résulte du défrichement des zones humides, qui étaient moins convoitées par les agriculteurs entre 1986 et 2001 en raison de la disponibilité des terres dans les classes d'occupation du sol comme les savanes boisées et les forêts. Les surfaces de savanes arborées ont régressé de plus de $50 \%$ de leur superficie. Elles sont passées de 116820 ha en 2001 à 58 594 ha, soit près de $20 \%$ du taux de couverture au sol, en 2016. Cependant, les savanes boisées et les forêts claires ont conservé les mêmes proportions de décroissance que les savanes arborées. De 118957 ha en 2001, la savane boisée est passée à 55722 ha en 2016, soit environ $18 \%$ de la couverture végétale du département de Dianra. Contrairement aux surfaces végétales naturelles, les activités anthropiques ont connu une forte croissance. Les vergers d'anacardiers qui occupaient une superficie de 1117 ha, soit $0.36 \%$ de la superficie du département en 2001, sont passés en 2016 à 59214 ha, ce qui correspond à près de $20 \%$ de l'espace total du département. Ils sont présents dans presque tout le département avec des tailles d'exploitation importantes. Durant la même période, les autres espaces agricoles sont passés de 38006 ha, soit $12 \%$ du département, à 89993 ha, soit près de $30 \%$ de ce même espace.

L'extension spatiale des zones agricoles est liée à l'introduction de la culture de l'anacardier dans le département de Dianra. L'anacardier est un arbre fruitier de la famille des Anacardiaceae. Sa taille moyenne est comprise entre 6 et 8 mètres de hauteur. Ils sont cultivés par les populations pour son fruit (l'anacarde ou la pomme de cajou). C'est une plante qui s'adapte à tous types de 
sols, exceptés les sols hydromorphes. En Côte d'Ivoire, la culture de l'anacardier s'est principalement développée dans la zone de savane, moins favorable à la culture du cacao dont le pays est le premier producteur mondial. Cette défaveur bioclimatique de la zone des savanes en rapport avec la production du cacao a permis de conserver une grande partie de la végétation naturelle, là où le sud du pays est dominé par des plantations de cacaoyers.

Vers la fin des années 1990 l'introduction de la culture de l'anacardier dans cette zone par l'État par l'entremise de la SODEFOR, a eu un profond impact sur l'occupation du sol. Cette monoculture va connaître un développement rapide grâce à la mise en place des mécanismes d'achat de l'anacarde et de l'organisation des producteurs par l'État au début des années 2000.

En l'espace de quelques décennies, les populations du nord de la Côte d'Ivoire vont convertir les terres disponibles en vergers d'anacardiers, lesquels sont moins sensibles aux effets des changements environnementaux et plus rentables économiquement que la culture du coton et les cultures vivrières,

Aujourd'hui, la Côte d'Ivoire, constitue l'un des principaux pays producteurs de l'anacarde. La majorité de sa production est exportée vers l'Asie pour la consommation de la menthe et différents usages dans l'industrie médicale et cosmétique.

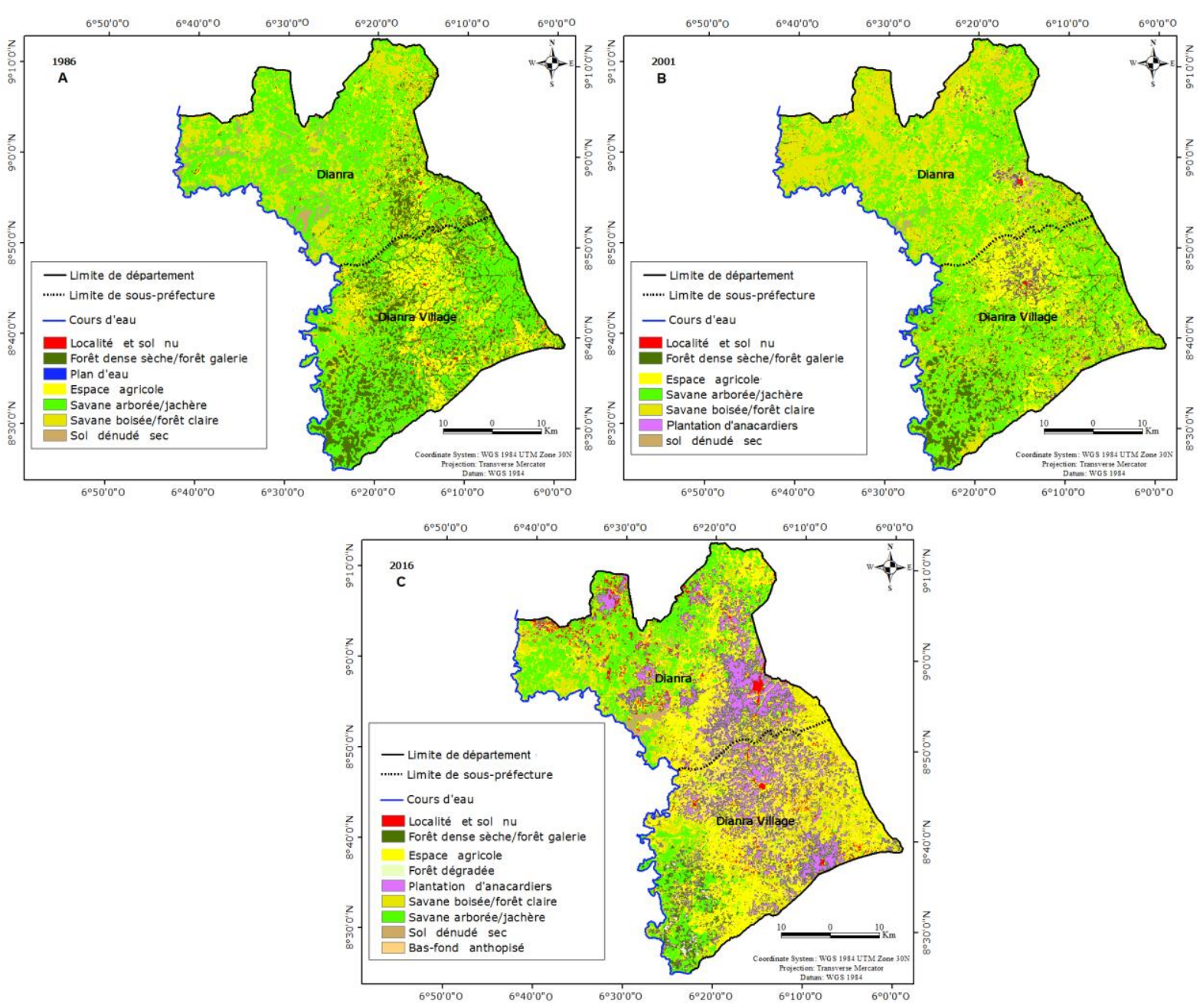

Figure 4 : Classifications, occupation du sol en 1986 (A), 2001 (B) et 2016 (C)

(Source : Images TM (1986), ETM+ (2001) et OLI (2016)) 


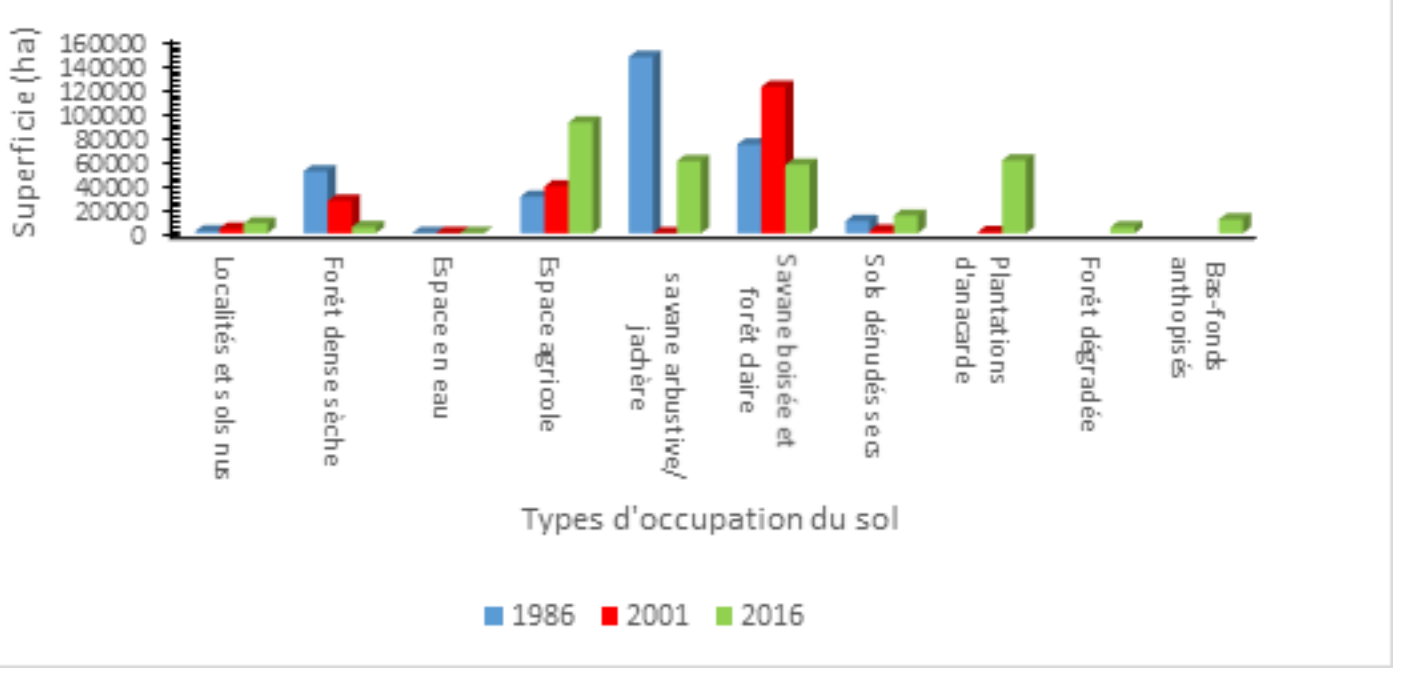

Figure 5 : Dynamique spatiale des types d'occupation du sol de 1986 à 2016 [source : Landsat 1986, 2001 et 2016]

La Figure 5 traduit les tendances de la dynamique d'occupation spatiale de Dianra entre 1986 et 2016 . Elle met en évidence deux grandes phases de l'évolution de l'occupation des terres du département. La première phase, située entre 1986 et 2001, est marquée par une faible occupation du sol par les cultures et une plus grande couverture du sol par la végétation naturelle. La seconde phase, comprise entre 2001 et 2016, montre une inversion des tendances. On observe plutôt une baisse de l'occupation du sol par la végétation naturelle, à l'exception de la classe savane arbustive et jachère qui connait une hausse liée à la multiplication des surfaces de jachère, et une augmentation des surfaces agricoles.

\subsection{Conflits d'usages liés à la dynamique spatiale dans le département de Dianra}

Par définition, le conflit est une lutte entre deux ou plusieurs personnes. II implique toujours, quelles que soient ses origines, ses objectifs et son déroulement, une opposition ou un antagonisme entre des catégories d'acteurs aux intérêts momentanément ou définitivement divergents (Kouadio, 2015).

Les conflits identifiés dans le département de Dianra relèvent assurément de logiques économiques, mais aussi identitaires. Les groupes d'acteurs qui interagissent dans l'espace l'investissent de significations différentes et, par conséquent, d'usages différents. A Dianra, deux principaux types de conflits ont été identifiés : les conflits entre autochtones (agriculteurs) et allochtones (éleveurs), et les conflits inter-villageois principalement liés à la délimitation des terroirs villageois.

\subsection{1- Les types de conflits}

\subsubsection{1- Les conflits entre autochtones (agriculteurs) et} allochtones (éleveurs)

Les conflits entre les agriculteurs et les éleveurs sont liés à l'accès aux ressources. Ils sont généralement déclenchés à la suite de dégâts de cultures et de désaccords sur les pistes à bétail. Les dégâts affectants les cultures (maïs, riz, igname, coton, anacarde, maraîchères) par les bœufs constituent des coûts supplémentaires dans l'activité agricole et mettent parfois en péril la sécurité alimentaire des agriculteurs ayant subi les dommages (cf. Figure 6).

Avec l'accroissement des vergers d'anacardiers (cf. Figure $4 C$ ), une culture pérenne qui immobilise les terres durant plusieurs années, l'alternance de l'agriculture et de l'élevage disparaît pour faire place à la monoculture. En effet, avant l'adoption de l'anacarde, les éleveurs disposaient de grandes surfaces de vaine pâture, qui permettaient de faire paître librement le bétail en dehors de leurs terres, sur les bords des chemins, les friches, les " terres vaines et vagues », sur l'ensemble des terres après la récolte. Avec le développement de l'anacarde, les espaces de libres accès sont devenus de plus en plus rares et difficiles d'accès pour les éleveurs à cause de l'empiètement des zones agricoles. Selon les dires du chef des éleveurs de Dianra :

"Avant l'arrivée de l'anacarde à Dianra ici, les bœufs se promenaient partout. Tu peux même faire un an ou deux ans sans problème de dégât, mais aujourd'hui c'est chaque jour qu'il y a des dégâts. Partout où nous passons, on trouve des champs. De Yélétiélé jusqu'à Faraba (cf. Figure 2) tout est devenu champs d'anacarde, nous ne savons plus par où passer ".

En effet, les campagnes de l'anacarde coïncident généralement avec les périodes de transhumance et rendent les conflits quotidiens. Selon les parties en conflit, les dégâts occasionnés sur les récoltes ou les cultures (figure 6) s'expliquent par l'amenuisement des terrains de parcours et des espaces de pâturage, conséquence des changements de l'occupation du sol engendrés par l'essor de la culture de l'anacarde.

Le relief de la localité de Dianra, constitué pour l'essentiel de plateaux de faible altitude, offre une uniformité favorisant la circulation du bétail dans un espace où l'élevage extensif constitue depuis les années 1970 une des composantes majeures des systèmes de production. 
Le développement de l'anacarde est donc devenu un enjeu capital dans l'économie agricole du département. Sa culture suscite une véritable « course à la terre". Cette dynamique oblige les pasteurs dont le système est fondé sur l'exploitation extensive des parcours naturels à s'adapter à la nouvelle donne foncière. De ce point de vue, les institutions sociales chargées de réguler l'accès aux ressources se sont constitués non pas à partir d'un souci partagé de parvenir à des gains communs grâce à la coopération, mais plutôt d'un conflit politique et d'une négociation stratégique entre agriculteurs et éleveurs.

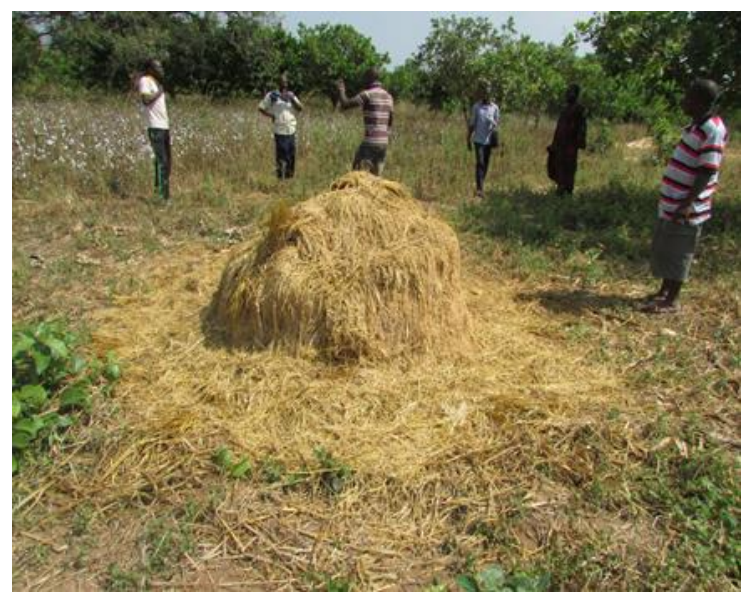

Figure 6 : Récolte détruite à Faraba (Cliché : Amani $R$, 2016)

\subsubsection{2- Les conflits inter-villages liés à la réduction des espaces et à l'essor de la culture de l'anacarde.}

Dans le département de Dianra comme dans la plupart des régions de la Côte d'Ivoire, les limites des terroirs villageois restent approximatives. Les enquêtes montrent que ces limites ont été définies suivant des repères qui sont de nature variable, et peuvent prendre la forme de marigots, de sommets de colline, de mares, de puits ou même d'arbres caractéristiques (manguiers, baobabs, etc.). Selon les mythes fondateurs, ces limites auraient été déterminées par la rencontre entre deux membres de groupes de villages voisins à l'occasion de chasses et de campagnes de défrichement. Cette matérialisation coutumière des limites entre les villages était en quelque sorte acceptée par les villageois à cause de l'abondance des ressources. Avec la réduction des espaces de culture disponibles, les désaccords latents entre les villages refont surface et occasionnent des conflits ouverts entre certains villages (par exemple, ceux de Faraba et de Léssimasso) qui se disputent des terres.

\subsection{2- Les éléments structurants des conflits à Dianra}

Le conflit est avant tout un processus social. On peut cependant décrire la matérialité des objets et des espaces qui motivent l'engagement des différents protagonistes. Les causes des conflits d'usage dans le département de Dianra s'inscrivent au sein de deux types d'espaces: l'espace des objets et des activités contestées, et l'espace des ressources protégées. Le premier type révèle la nature des usages et activités rejetés, alors que le second révèle les enjeux territoriaux en matière de gestion des ressources.

\subsubsection{1- L'établissement de barrières limitant la circulation du bétail : objets de conflits}

Un des moyens les plus immédiats est d'agir directement sur l'organisation spatiale afin de s'opposer à un usage ou à une activité. Ainsi, les conflits d'accès donnent régulièrement lieu à l'édification de barrières (cf. Figure 7) ou à la multiplication de signaux matérialisant le périmètre d'occupation agricole. À Dianra, les actions sur l'espace physique sont nombreuses, et assez significatives. On y distingue les actions spécifiquement dédiées à la régulation de la circulation des troupeaux, les actions de réaménagement des berges des barrages et enfin les actions de dégradations des cultures, qui laissent des traces visibles des confrontations entre les acteurs. Ces trois types d'actions visant à s'opposer à un usage du sol, ont des impacts durables ou temporaires sur le paysage. On citera comme exemple la formation de buttes de terres et de fossés aux abords des champs ou des jachères, qui ont pour objectif d'empêcher la circulation du bétail local et des transhumants.

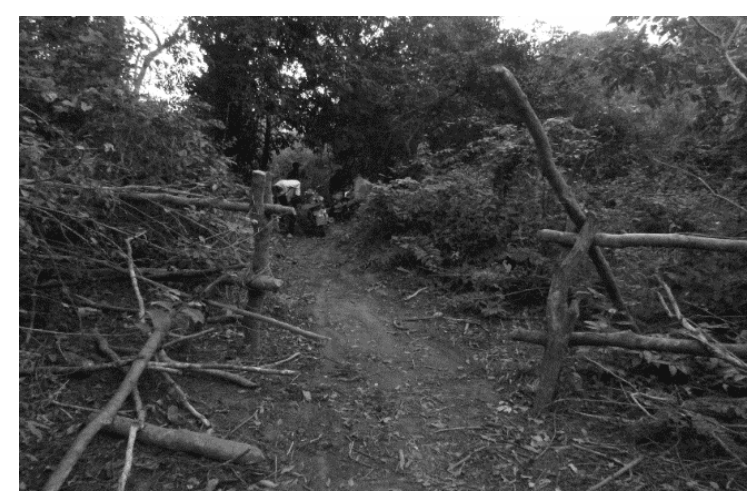

Figure 7 : Clôture de fortune érigée pour empêcher le passage des troupeaux (Cliché : AMANI R, 2016)

\subsubsection{2- Les forêts classées : espace de compétition} entre agriculteurs et éleveurs

Les seconds types d'objets spatiaux à l'origine du déclenchement des conflits entre agriculteurs et éleveurs dans le département de Dianra sont les forêts classées, à savoir la forêt classée de Kani Bandama rouge (Est) et celle de la Kowa (cf. Figure 2). Ces forêts classées témoignent de l'image de la saturation de l'espace dans le département de Dianra. Les éleveurs s'y infiltrent à cause du manque d'espace pour leurs activités dans le domaine rural. Pour les éleveurs, ces forêts classées constituent un réservoir de ressources important pour leurs activités à cause de la disponibilité des ressources naturelles (l'eau et le fourrage). Cependant, les éleveurs ne sont pas les seuls à occuper ces forêts classées. Outre les éleveurs, on note également la présence de colons agricoles à l'intérieur de ces forêts classées, qui se sont installés pour faire de l'agriculture (cf. Figure 4C) (plantation cacaoyère, plantation d'anacardier, coton et des cultures vivrières (mais, manioc, banane, du riz... etc.) et même la création de campements (cf. Figure 8 et 9). 


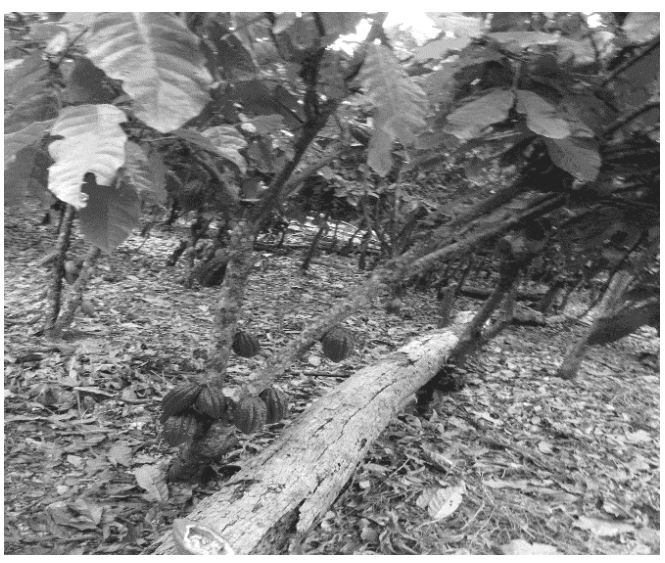

Figure 8 : plantation de cacaoyers dans la forêt classée de Kani Bandama rouge (Cliché AMANI R, 2016).

L'infiltration massive des éleveurs et des agriculteurs dans les forêts classées du département de Dianra commença à partir des années 2002, à la suite de la crise militaro-politique qu'a connue la Côte d'Ivoire. Cette crise a fragilisé la légitimité de l'État ivoirien dans la zone Nord sous contrôle des rebelles. Les forêts classées qui étaient des espaces mis en défense ont perdu leur protection et sont devenues l'objet de convoitise de la part des agriculteurs et des éleveurs. Éleveurs et agriculteurs jugeaient leur présence légitime à l'intérieur de ces forêts classées. Ces derniers affirment avoir été installés dans ces forêts par des autochtones se prétendant propriétaires du terrain de ces espaces domaniaux.

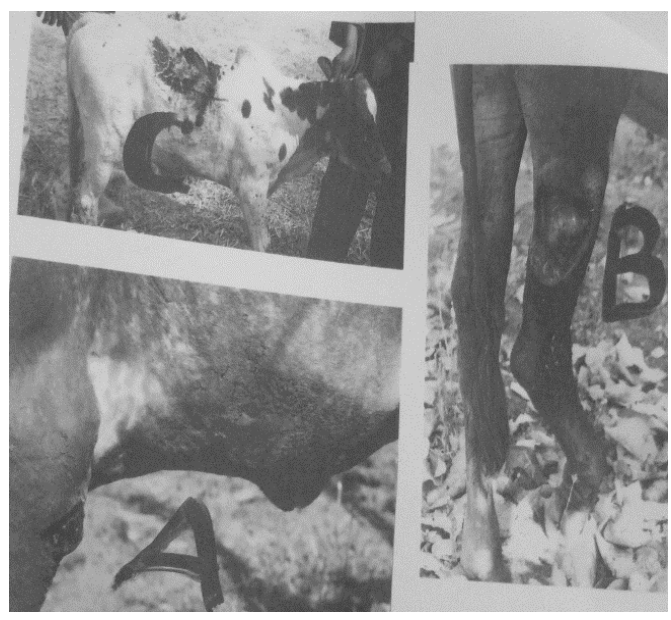

Figure 10 : Bœufs tailladés à la machette lors d'un conflit dans la forêt classée de Kani Bandama rouge [Cliché : MIRAH de Dianra,

Le lieu du déroulement de ces différentes confrontations entre les agriculteurs et les éleveurs est révélateur de la saturation de l'espace rural. Les dernières ressources naturelles (terres cultivables, eau et fourrage) étant disponibles dans les forêts classées, les agriculteurs et éleveurs se ruent vers ces espaces pour exploiter les ressources bien que cela soit interdit par l'État.

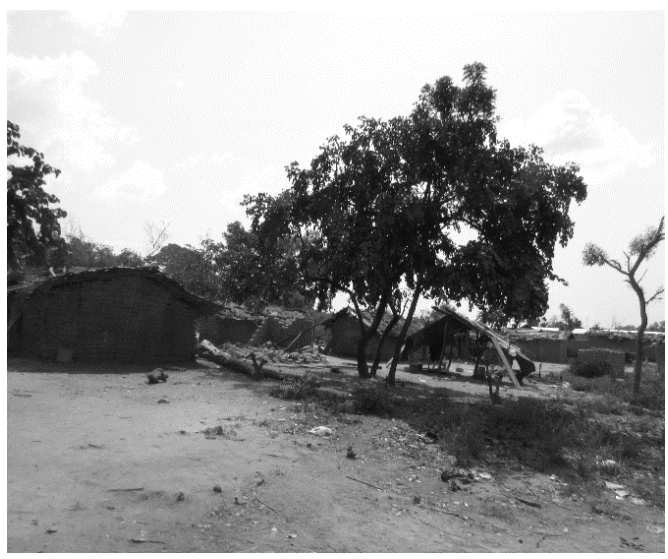

Figure 9 : Campement de Seydoukaha 2 dans la forêt classée de Kani Bandama rouge (Cliché: AMANI R, 2016)

Les éleveurs considèrent leur présence dans les forêts classées comme légitime, dans la mesure où les administrateurs des forêts classées cautionnent leur présence au moyen d'un ticket d'accès. Selon eux, cette situation se justifierait ainsi par des arrangements passés avec l'administration forestière. Dès lors, ils font paître les troupeaux sur tous les périmètres des forêts classées.

Cette double présence d'agriculteurs et d'éleveurs inégalement installés dans les forêts classées est la source d'importants conflits survenus dans le département de Dianra, qui se soldèrent par plusieurs blessés et la destruction des troupeaux (cf. Figure 10 et 11).

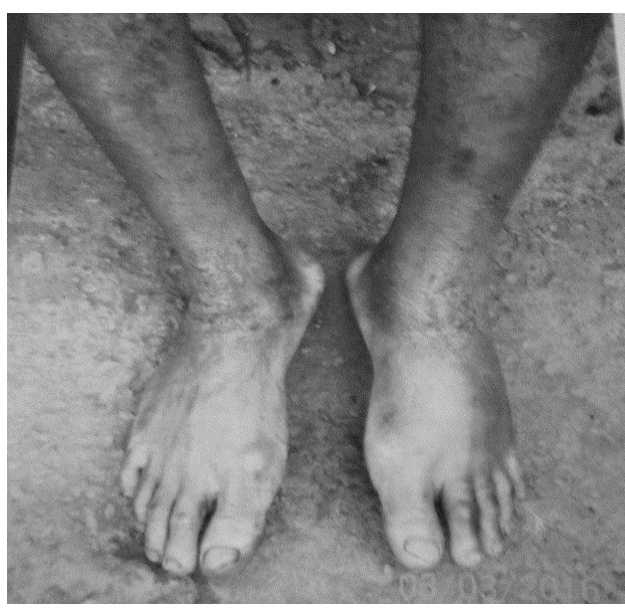

Figure 11 : Bouvier battu par les colons agricoles de la forêt classée de Kani Bandama rouge [Cliché : MIRAH de Dianra, 2016]

\subsubsection{3- Les conflits liés aux barrages agropastoraux}

Les conflits observés autour des barrages agropastoraux (cf. Figure 12) dans le département de Dianra relèvent de différentes causes. La première est celle de la croissance rapide du cheptel bovin local. 


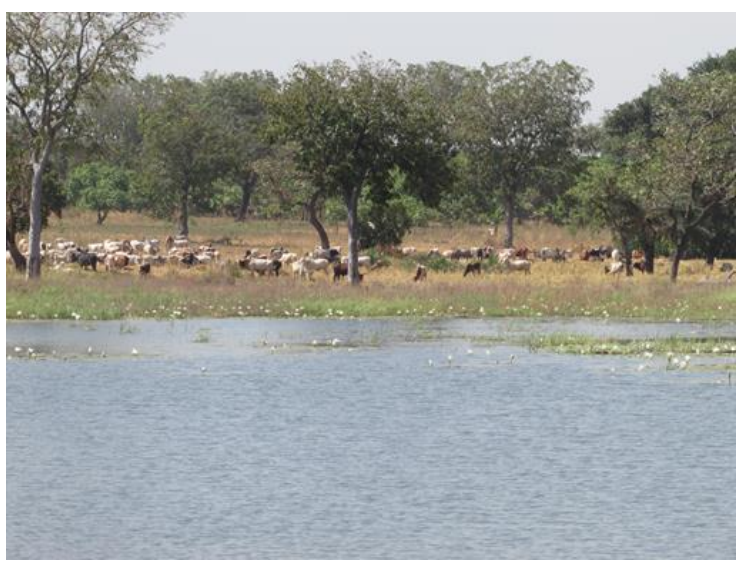

Figure 12 : Troupeau aux abords d'un barrage agropastoral à Yélétiélé
En effet, selon les statistiques du ministère des Ressources animales et halieutiques (MIRAH), le cheptel bovin est passé de 31367 têtes de bœufs en 2014, à 36 032 têtes de bœufs en 2015, ce qui correspond à une augmentation de l'ordre de $15 \%$. Cette rapide augmentation du cheptel n'a pas donné lieu à la multiplication des points d'eau. Ainsi, l'insuffisance des abreuvoirs pour le bétail, a suscité des déplacements massifs des troupeaux aux abords de deux barrages agropastoraux, plus précisément les barrages de Yélétiélé et de Dianra, dans le département. De plus, la dynamique de l'occupation du sol a entrainé la mise en culture des espaces périphériques des barrages (cf. Figure 13). La non-formalisation de couloirs d'accès aux barrages a entraîné beaucoup de débordement des troupeaux dans les champs. Cela a engendré de multiples dégâts aux cultures avec son corollaire de conflits.

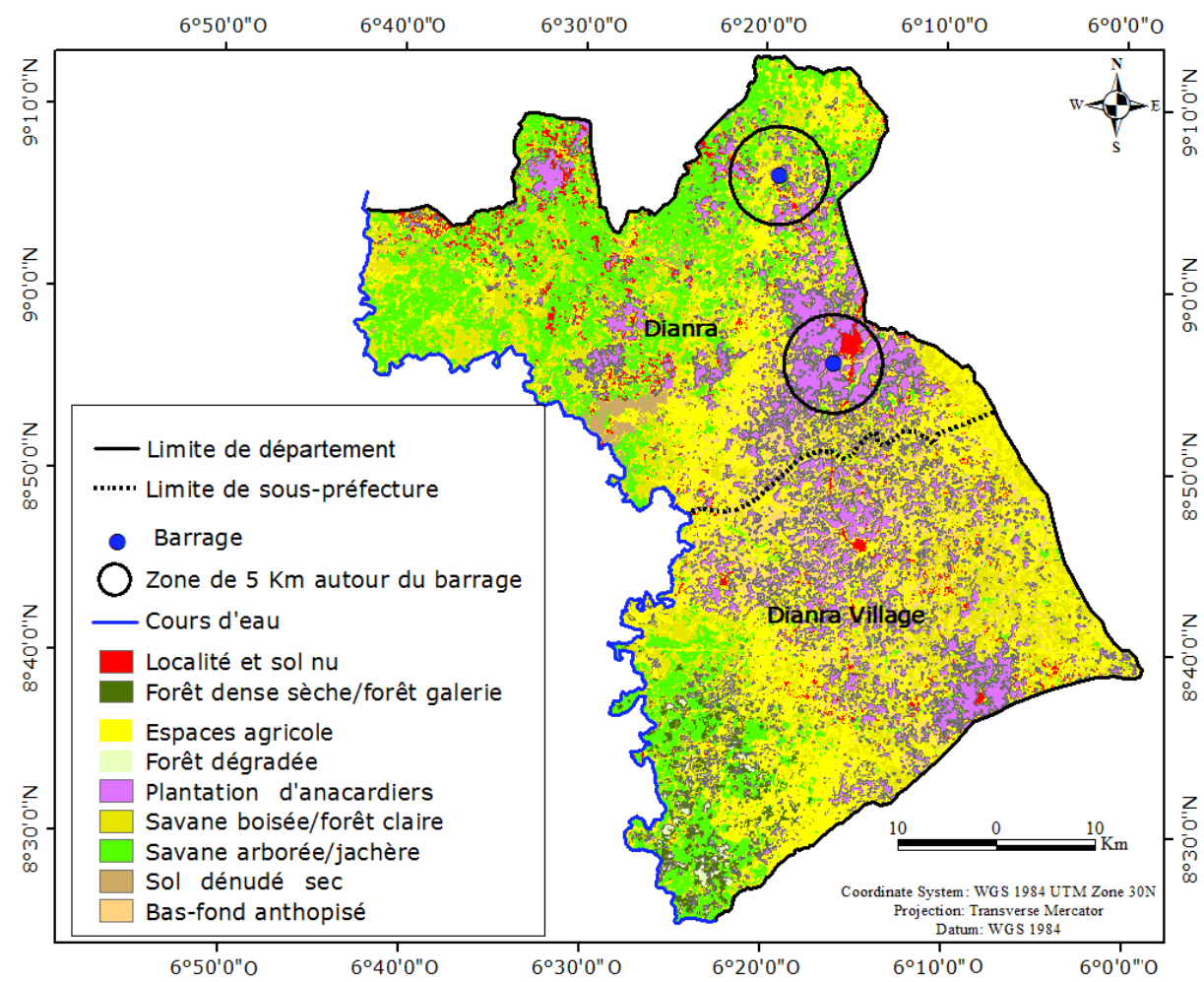

Figure 13 : Classification occupation du sol et emprise agricole autour des barrages dans le département de Dianra en 2016 [Source : Landsat, OLI/ TIRS, 2016]

\section{Discussion}

\subsection{Télédétection et cartographie de l'occupation du sol}

L'utilisation des techniques de la télédétection dans cette étude a permis de réaliser la cartographie spatiotemporelle de l'occupation du sol dans le département de Dianra. La télédétection se révèle être un outil remarquable dans l'évaluation et le suivi des types d'occupation du sol.

L'algorithme de «maximum de vraisemblance » utilisé pour la classification a permis dans l'ensemble d'aboutir à des résultats satisfaisants. En effet, la classification des images donne des précisions globales estimées à 93,50\% (1986), 96,18\% (2001) et 93,94\% (2016). Ces indices de précision globaux sont acceptables, car ils sont nettement au-dessus du seuil de référence des 80\% requis par Girard et Girard (2010).

Ces précisions globales de classification sont similaires à celles obtenues par Soro et al. (2013), dans une étude réalisée sur la zone de savane soudanienne ivoirienne. Les résultats globaux de précision des classifications obtenus à la suite de cette étude sont de $97 \%$ et $98 \%$ respectivement, pour les années 1986 et 2000 . II en est de même pour Kouassi (2014), qui a trouvé des précisions globales de $96,20 \%$ en $1987,95,03 \%$ en 2002 et $94,80 \%$ en 2012 , lors d'une étude réalisée sur la 
dynamique d'occupation du sol en milieu de savane. Cette bonne qualité des précisions globales des classifications s'explique en partie par le fait que l'acquisition des images s'est faite en saison sèche, durant laquelle les perturbations dues à la couverture nuageuse sont quasiment absentes.

De même, les coefficients Kappa obtenus à la suite des classifications des images sont supérieurs à $80 \%$. Ces résultats sont classés excellents, selon Cunningham, (2009). En effet, Cunningham a classé le coefficient Kappa des classifications à trois niveaux, selon les scores obtenus : excellent quand il est compris entre 0,81 et 1 bon lorsqu'il est compris entre 0,61 et 0,80 et modéré lorsqu'il entre 0,21 et 0,60 . Nos résultats respectent le principe de Pontius (2000) selon lequel, une étude de l'occupation des sols peut être validée si l'indice Kappa est au moins compris entre 0,5 et 0,75 .

Cependant, si l'algorithme utilisé donne des résultats globalement satisfaisants, il présente aussi des insuffisances. En effet, les campagnes de terrain ont révélé des confusions entre certaines classes d'occupation du sol. Il s'agit d'abord des habitats/sols nus et des espaces agricoles. Cette situation pourrait s'expliquer par le fait que les images ont été prises en saison sèche, durant laquelle la végétation, pour la plupart herbacée, située entre les plants des cultures annuelles, a tendance à sécher (sans activité chlorophyllienne) pour se confondre avec les sols nus. Cette même remarque a été faite par Soro et al. (2013) dans une étude basée sur la classification d'image satellite au nord de la Côte d'Ivoire.

\subsection{Dynamique des types d'occupation du sol dans le département de Dianra}

Les cartes et les calculs statistiques produits ont permis de mesurer l'évolution spatiale des différents types d'occupation du sol dans le département de Dianra. La dynamique des types d'occupation du sol en général et de la couverture végétale en particulier est faible entre 1986 et 2001. Ainsi, de 1986 à 2001, les dynamiques observées au niveau de la végétation étaient de - $4 \%$ pour les forêts denses sèches, $-1 \%$ pour les savanes boisées et $+34 \%$ pour les savanes arbustives. Ces résultats contredisent ceux des études menées dans le sud de la Côte d'Ivoire à la même période, à cause de l'impact négatif que la culture du café et du cacao ont eu sur les forêts. Cela est signifié par Konan (2009), dans la région d'Oumé, à propos de la dynamique des formations agroforestières en Côte d'Ivoire. En revanche, entre 2001 et 2016, le département de Dianra a subi une perte considérable des surfaces de végétations naturelles, à cause de l'introduction, à partir de 1990, de la culture de l'anacarde, qui offrit des opportunités économiques aux populations. Ainsi, les superficies de vergers d'anacardiers sont passés en 15 ans de 1116,99 ha en 2001, à 59214 ha en 2016, alors que les forêts claires et les savanes arborées ou boisées sont passés respectivement de 118957 et 116820 ha en 2001 à 55703 et 58594 ha en 2016.

\subsection{Conflits liés à la pression spatiale}

Les situations de conflits entre les agriculteurs et les éleveurs dans le département de Dianra sont dues à la raréfaction des ressources naturelles. L'analyse du déroulement des conflits et des espaces qu'ils occupent témoigne des effets sous-jacents des mutations institutionnelles (comme les règles d'accès aux ressources). Leur émergence relève des changements des pratiques et de l'occupation concurrentielle de l'espace entre des acteurs d'intérêt divergent. Les espaces de compétition et de conflits entre les différents protagonistes concernent aussi bien le domaine rural que les espaces domaniaux (forêts classées). Cette pression sur l'espace est une des conséquences de la crise militaro-politique de 2002, qui a favorisé la modification des règles sociales d'appropriation et d'usage des ressources naturelles.

A Dianra, nos analyses montrent que plus les pâtures se raréfient, plus les tensions entre les acteurs sont importantes, à cause de la proximité des champs et des pâtures. Trianneau, (2012) donne ce même problème du manque de limites entre espaces à usage agricole et espaces à usage pastoral comme un facteur participant à la survenue des conflits entre agriculteurs et éleveurs.

Dans le département de Dianra, le manque d'espace spécifique consacré aux activités agropastorales et l'emprise des zones agricoles sur les points d'eau dédiés au bétail rendent donc difficile la pratique de l'élevage traditionnel et contribuent à entretenir les conflits entre éleveurs et agriculteurs.

\section{Conclusion et Perspectives}

L'analyse de l'évolution de l'occupation du sol de l'espace territorial du département de Dianra est basée sur des images satellites Landsat TM de (1986), ETM+ de (2001) et OLI/TRIS de (2016). Les résultats des traitements qui ont été validés à partir de la matrice de confusion ont permis d'obtenir des précisions globales de $94 \%$ (1986), $97 \%(2001)$ et $94 \%$ (2016). Les résultats cartographiques ont permis d'appréhender les formes spatiales et la dynamique des différents types d'occupation du sol. Ainsi, de 2001 à 2016, des espaces dédiés aux cultures vivrières ont été convertis en plantation d'anacardiers, de même que l'ont été $29 \%$ des savanes et $8 \%$ des forêts denses sèches et des forêts galeries.

L'occupation de l'espace va connaître une dynamique accélérée avec le développement de la culture de l'anacarde. L'amenuisement progressif des terres agricoles et des pâturages va perturber les rapports entre agriculteurs et éleveurs, ce qui aboutit souvent à des conflits. Ces conflits sont devenus fréquents et se traduisent par des affrontements violents soldés par des dégâts matériels et des pertes en vie humaine.

Ainsi, l'accès aux ressources s'inscrit dans une dynamique d'interaction mobilisant des stratégies diverses de contrôle et de maintien de l'accès aux ressources : changement des règles du jeu par la mise en place de nouvelles règles, des arrangements, manipulation de l'identité ou du référent professionnel.

L'État, qui a pris la pleine mesure de la situation, met en place des programmes de sécurisation des territoires fonciers ruraux afin de renforcer la cohésion sociale. Ainsi, il exécute un programme de délimitation des territoires de chaque village avec le soutien financier de l'Agence française pour le développement (AFD). L'objectif de ce programme vise à contribuer à la réduction des conflits entre les différents villages, en permettant à chaque village de connaître officiellement ses limites territoriales. Aussi, la délimitation des territoires des villages vise à terme à faciliter celle des parcelles de terres. Pour rappel, les conflits entre villages font partie intégrante du paysage des conflits dans le domaine du foncier rural

Au terme de cette étude, plusieurs questions restent sans réponse : quel est l'avenir de l'élevage face à la réduction 
des pâturages et aux changements institutionnels? Comment peut-on garantir la sécurité alimentaire face à l'occupation des espaces de culture vivrière par les plantations d'anacardier? Pour la suite de nos travaux, nous souhaiterions faire une modélisation des changements institutionnels et de la diffusion spatiale de l'anacarde par la simulation multi-agents.

\section{Remerciements}

Ce travail qui a nécessité un important investissement en temps et en argent n'aurait pu aboutir sans le soutien de personnes et d'institutions à qui nous tenons à exprimer ici notre vive reconnaissance.

Nous tenons à exprimer notre gratitude à l'Université Félix Houphouët-Boigny et particulièrement aux enseignants de l'IGT (Institut de géographie tropicale).

Merci au Dr FOKOU Gilbert, chercheur au Centre suisse de recherches scientifiques en Côte d'Ivoire pour nous avoir donné l'opportunité d'intégrer le projet "Analyse des effets des transformations institutionnelles sur les systèmes socio-écologiques en vue de définir les stratégies de sécurisation des moyens d'existence des petits producteurs ".

\section{Références}

Avenard J.M., Eldln M., Girard G., Sircoulon J., Touchebeuf P., Guillaumet J.L., Adjanohoun E., Perraud A., 1971. Le milieu naturel de la Côte d'Ivoire. Les mémoires de l'ORSTOM, Paris, pp.82-84

Brou Y. T., Chaléard J.-L., 2007. Visions paysannes et changements environnementaux en Côte d'lvoire. Annales de géographie, Armand Colin pp. 65-87.

Corniaux C., Thebaud B. et Gautier D., 2012. La mobilité commerciale du bétail entre le sahel et les pays côtiers : l'avenir du convoyage à pied, Nomadic Peoples, Volume 16, №.2, pp. 6-25

Cunningham M., 2009. More than just the kappa coefficient : a program to fully characterize inter-raté reliability between two raters. SAS global forum, Vol. 2009, $242 \mathrm{p}$

Brown, D. G. et Duh, J.-D., 2004. Spatial simulation for translating from land use to land cover. International Journal of Geographical Information Science, 18(1), pp. $35-60$

Diallo H., Bamba I., Barima Y. S. S., Visser M., Ballo A., Mama A., Vranken I., Maiga M., Bogaert, J., 2011. Effets combinés du climat et des pressions anthropiques sur la dynamique évolutive de la végétation d'une zone protégée du Mali (Réserve de Fina, Boucle du Baoulé). Science et changements planétaires/ Sécheresse, 22(2), pp. 97-107.

Dugue P., Koné F. R., Koné G. et Akindes F., 2004. Production agricole et élevage dans le centre du bassin cotonnier de Côte d'Ivoire : développement économique, gestion des ressources naturelles et conflits entre acteurs. Cahiers Agricultures, 13 (6) pp. 504-509

Dusseux P., 2014. Exploitation de séries temporelles d'images satellites à haute résolution spatiale pour le suivi des prairies en milieu agricole. Phd thesis. Université Rennes 2, 283 p. https://tel.archivesouvertes.fr/tel-01131770/document

Fotsing E., 2009. SMALL Savannah : Un système d'information pour l'analyse intégrée des changements d'utilisation de l'espace à l'extrême nord du Cameroun. (S.I.) : Universiteit Leiden. Pp.16-21.

Girard M.-C., Girard C.M., 2010. Traitement des données de télédétection-2e éd.: Environnement et ressources naturelles. (S.I.) : Dunod, $576 \mathrm{p}$.

Institut National de la Statistique (INS), 2014. Recensement Général de la Population et de l'Habitat 2014 (RGPH 2014), Abidjan.

Jobin B., 2007. Changements de l'occupation du sol dans le sud du Québec pour la période 1993-2001. (S.I.) : Service canadien de la faune. $325 p$

Konan D., 2009. Impact de la cacaoculture sur la flore et la végétation en zone de forêt dense humide semiedécidue : cas du Département d'Oumé (Côte d'lvoire). Mémoire de DEA, UFR Biosciences, Univ. CocodyAbidjan. $95 \mathrm{p}$

Konan K. E., 2008. Conservation de la diversité végétale et activité humaine dans les aires protégées du sud forestier ivoirien : L'exemple du Parc National d'Azagny. Université de Cocody-IGT 278 p.

Kouadio N. K. F., 2015. Conflits autour de l'exploitation et de la gestion des lagunes ivoiriennes: cas du secteur est de la lagune ébrié. Revue de Géographie Tropicale et d'Environnement, 1(1), pp.79-89.

Kouassi, J.-L., 2014. Suivi de la dynamique de l'occupation du sol à l'aide de l'imagerie satellitaire et des Systèmes d'Informations Géographiques: Cas de la Direction Régionale des Eaux et Forêts de Yamoussoukro (Côte d'Ivoire). (S.I.): (s.n.).https://doi.org/10.13140/2.1.2359.7128

Landais E., 1994. Système d'élevage. D'une intuition holiste à une méthode de recherche, le cheminement d'un concept-In: C. Blanc-Pamard et J. Boutrais, pp.1549.

Mahop F., 1983. Etude pédologique et représentation cartographique à 1/10 000 d'une zone représentative des savanes du centre-nord-ouest de la Côte d'lvoire (partie nord de la coupure IGN de Mankono à 1/200 000 sous-préfecture de Dianra). Abidjan :ORSTOM http://www.documentation.ird.fr/hor/fdi:010027395

Oszwald J., Lefebvre A., Arnauld de Sartre X., Thales M., Gond V., 2010. Analyse des directions de changement des états de surface végétaux pour renseigner la dynamique du front pionnier de Maçaranduba (Brésil) entre 1997 et 2006. Télédétection, 9(2), pp.97-111.

Pontius Jr. R. G., 2000. Quantification error versus location error in comparaison of categorical maps. Photogrammetric Engineering and remote Sensing, 66(8) : pp.1011-1016.

Sokouri D. P., Yapi-Gnaoré C. V., N'Guetta A. S. P., Loukou N. E., Kouao B. J., Gnénékita Touré G., Sangaré A. et Kouassi A., 2009. Utilisation et gestion des races taurines locales sous la pression des croisements avec les zébus dans les régions Centre et Nord de la Côte d'Ivoire. Journal of Animal \& Plant Sciences, 5(2), pp.456-465.

Soro T., Kouahou D. B., Kouassi A. E., Soro G., Kouassi A. M., Kouadio K. E., Yéo M-S., Soro N., 2013. Hydroclimatologie et dynamique de l'occupation du sol du bassin versant du HautBandama à Tortiya (Nord de la Côte d'lvoire) [VertigO]http://www.erudit.org/en/journals/vertigo/2013v13-n3 vertigo01538/1026870ar/abstract/ 
Trianneau K. N. B., 2012. Dynamiques spatiales et mobilités paysannes : les relations agriculture/ élevage dans deux terroirs agro-pastoraux du Delta intérieur du fleuve Niger (Mali). Université de Poitiers. $356 \mathrm{p}$

\section{Titre et Légendes en anglais}

CONTRIBUTIONS OF LANDSAT IMAGES TO THE DYNAMICS OF LAND COVER AND ANALYSIS CONFLICTS USE IN THE DIANRA DEPARTMENT IN NORTHERN CÔTE D'IVOIRE

Figure 1: Cashew orchard in Dianra

Figure 2: Presentation of Dianra department: [Source: BNETD, 2014]

Figure 3: Landsat 5 and 7 (Red = band 2, Green = band 4 , Blue $=$ band 6$)$ and Landsat $8($ Red $=$ band 3 , Green $=$ band 5 , Blue $=$ band 7 ) image extracts.

Figure 4: Land cover in 1986 (A), 2001 (B) and 2016 (C) (Source: TM (1986), ETM+ (2001) and OLI (2016) images)

Figure 5: Spatial dynamics of land cover types from 1986 to 2016 [source: Landsat 1986, 2001 and 2016].

Figure 6: Destroyed crop in Faraba (Photo: Amani R, 2016)

Figure 7: Makeshift fence erected to prevent the passage of herds (Photo: AMANI R, 2016)

Figure 8: Cocoa tree plantation in the Kani Bandama red classified forest (Photo: AMANI R, 2016).

Figure 9: Seydoukaha 2 camp in the Kani Bandama red forest (Photo: AMANI R, 2016)

Figure 10: Oxen slashed with machetes during a conflict in the Kani Bandama red forest [Photo: MIRAH de Dianra],

Figure 11: Cattle beaten by agricultural settlers in the Kani Bandama red forest [Photo: MIRAH de Dianra, 2016].

Figure 12: Herd on the edge of an agro-pastoral dam in Yélétiélé

Figure 13: Land cover and agricultural holdings around dams in Dianra Department in 2016 [Source: Landsat, OLI/ TIRS, 2016].

Table 1: Characteristics of the Landsat spectral bands of the satellite images used

Table 2: Description of the different types of land use retained

Table 3: Confusion matrix of the 1986 image (in\%)

Table 4: Confusion matrix for the 2001 image (in\%)

Table 5: Confusion matrix of the 2016 image in percentage (\%) 